\title{
Short communication: Roles of outer membrane protein W on survival, cellular morphology, and biofilm formation of Cronobacter sakazakii in response to oxidative stress
}

\author{
Xiyan Zhang, ${ }^{1,2 *}$ Jina Gao, ${ }^{1 *} \mathrm{Na}$ Ling, ${ }^{2 *}$ Haiyan Zeng, ${ }^{2}$ Liaowang Tong, ${ }^{1}$ Maofeng Zhang, ${ }^{1}$ Jumei Zhang, ${ }^{2}$ \\ Qingping $\mathrm{Wu}^{2}{ }^{2} \dagger$ and $\mathrm{Ying}$ wang $\mathrm{Ye}^{1,2} \dagger$ \\ ${ }^{1}$ School of Food Science and Engineering, Hefei University of Technology, Hefei 230009, China \\ ${ }^{2}$ State Key Laboratory of Applied Microbiology Southern China, Guangdong Provincial Key Laboratory of Microbiology Culture Collection \\ and Application, Guangdong Institute of Microbiology, Guangzhou 510070, China
}

\section{ABSTRACT}

Cronobacter species are a group of opportunistic foodborne pathogens that cause rare but severe infections in neonates. Tolerance to environmental stress in Cronobacter is known; however, factors involved in oxidative stress are undefined. In this study, Cronobacter sakazakii survival, cellular morphology, and biofilm formation in response to oxidative stress were evaluated between the wild type (WT) and an outer membrane protein W $(O m p W)$ mutant. The survival rates of $\Delta O m p W$ strain after treatment with 1.0 and $1.5 \mathrm{~m} M$ hydrogen peroxide were significantly reduced compared with those of WT. Morphological changes, including cell membrane damage and cell fragmentation, in $\Delta O m p W$ were more predominant than those in WT. By crystal violet staining, we also observed increased biomass in $\Delta O m p W$ biofilms as compared with WT following treatment with 0.5 and $1.0 \mathrm{mM} \mathrm{H} \mathrm{H}_{2} \mathrm{O}_{2}$. Biofilms using scanning electron microscopy and confocal laser scanning microscopy further confirmed the structural changes of biofilms between $\mathrm{WT}$ and $\Delta O m p W$ in response to oxidative stress. The current findings show that $O m p W$ contributed to survival of planktonic cells under oxidative stress and the deletion of $O m p W$ facilitated the biofilm formation in C. sakazakii to adapt to oxidative stress.

Key words: Cronobacter sakazakii, outer membrane protein $\mathrm{W}(\mathrm{OmpW})$, oxidative stress, biofilm formation

\section{Short Communication}

Cronobacter species are important food-borne pathogens that cause rare but severe infections in newborns and adults (Holý and Forsythe, 2014; Ye et al., 2014). Powdered infant formula is the main transmission ve-

Received February 24, 2018.

Accepted October 15, 2018.

*These authors contributed to the manuscript equally.

†Corresponding authors: yeyw04@mails.gucas.ac.cn and wuqp203@ 163.com hicle of Cronobacter infections in newborns (Biering et al., 1989; van Acker et al., 2001), and tolerance or resistance to environmental stress in Cronobacter species have been reviewed (Gurtler et al., 2005).

Hydrogen peroxide has been widely applied to inactivate Escherichia coli O157, Listeria monocytogenes, and Salmonella (Huang and Chen, 2011; Back et al., 2014; Jiang et al., 2017), because no harmful residues remain following treatment in food engineering applications. Alvarez-Ordoñez et al., (2012) reported significant heterogeneity in RpoS activity and stress tolerance among natural isolates of Cronobacter sakazakii. Effects of genes, including $\operatorname{crt} X$, crtE, and $\operatorname{crt} Y$, involved in yellow pigmenting of C. sakazakii ES5 on oxidative stress have been described (Johler et al., 2010). Under conditions of stress, E. coli and related bacteria increase the expression of RpoS, and RpoS-dependent gene expression leads to general stress resistance of cells (Battesti et al., 2011). In E. coli, regulators including OxyR, SoxRS, and RpoS were involved in the resistance to oxidative stress (Chiang and Schellhorn, 2012). An RNA chaperone, hfq, has been found to contribute to the tolerance to oxidative stress in C. sakazkaii ATCC29544 (Kim et al., 2015). Expression of $O m p W$ is regulated by environmental factors such as oxidative stress in Salmonella and E. coli (Gil et al., 2007, 2009; Asakura et al., 2008; Morales et al., 2012).

We evaluated oxidative stress comparing the wild type (WT) C. sakazakii GDMCC1409C1 and an outer membrane protein $\mathrm{W}(\mathbf{O m p W})$ mutant. Construction of the OmpW mutant was previously described in Ling et al. (2018). In brief, 2 fragments flanking the targeted genes were amplified independently by PCR using primers containing the attB sequences (outside primers, W5-O: 5'-GGGGACAAGTTTGTACAAAAAAGCAGGCTACACTCCTTGGTTGGCAGAT-3' and W3-O: 5'-GGGGACCACTTTGTACAAGAAAGCTGGGTGCCTGCCTGAGCATTTCCTT-3') and linking sequences (inside primers, G5-I: 5'-GGTCCGGGTTCGCTATCTATACGATAAGTT TTTTCATTCC-3' and 
G3-I: $\quad$ 5'-ATAGATAGCGAACCCGGACCCGGGCTACCGCTTCTGAGTT-3') with genomic DNA as the template. The fragments were then joined together by fusion PCR using primers $\mathrm{W} 5-\mathrm{O}$ and $\mathrm{W} 3-\mathrm{O}$ as described (Ling et al., 2018). The resulting fusion homology arm was then purified and transformed into plasmid PHGM01 for recombination. Thereafter, the correct destination vector for mutagenesis was transferred by conjugation into C. sakazakii. The positive mutant was confirmed by sequencing the mutated regions.

To determine the survival of $C$. sakazakii cells, the enrichment cultures of $\mathrm{WT}$ and $\triangle O m p W$ were incubated in sterile Luria-Bertani broth (Luqiao, Beijing, China) with $0.5,1.0$, and $1.5 \mathrm{mM} \mathrm{H} \mathrm{H}_{2} \mathrm{O}_{2}$ (Sinopharm Chemical Reagent Co. Ltd., Shanghai, China) at $37^{\circ} \mathrm{C}$ for $1.0 \mathrm{~h}$ in the dark. Cronobacter sakazakii cells under oxidative stress were enumerated by plate counts on tryptic soy agar (Luqiao). Bacterial survival rates were calculated as the number of hydrogen peroxide-exposed cells divided by the initial cell number multiplied by $100 \%$. Oxidative stress survival was evaluated by 3 independent experiments. Based on normal distribution of data and homogeneity of variance, significant analysis of survival rates were performed using the Student's $t$-test statistical method with OriginPro 8.5.1 software (OriginLab, Northampton, MA).
From Figure 1A, we found that survival rates of

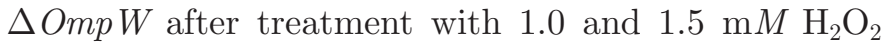
were significantly reduced $(P<0.05)$ compared with those of wild type. The findings might be related to reduction of OmpW expression or changes in the spatial structure of OmpW under oxidative stress. In enterohemorrhagic E. coli O157:H7, Asakura et al., (2008) demonstrated that the high level of $O m p W$ expression sensitized bacteria to oxidative stress. In Salmonella enterica serovar Typhimurium, Omp $W$ functions as an efflux channel for toxic compounds generated during oxidative stress (Gil et al., 2009). Gil et al., (2007) also demonstrated that $O m p W$ mediated efflux and resistance to paraquat in Salmonella Typhimurium and expression of $O m p W$ was inactivated by a superoxide. Morales et al., (2012) found that expression of $O m p W$ in Salmonella Typhimurium was negatively regulated on exposure to $\mathrm{H}_{2} \mathrm{O}_{2}$ and hypochlorous acid by response regulator AcrA, suggesting a negative correlation between expression of $O m p W$ and survival of cells.

To determine the cellular morphological changes of $C$. sakazakii under oxidative stress, the cells were harvested from the overnight culture. Cells were then treated with $0,0.5,1.0$, and $1.5 \mathrm{mM} \mathrm{H}_{2} \mathrm{O}_{2}$ for $1 \mathrm{~h}$ at $37^{\circ} \mathrm{C}$ with 180 $\mathrm{rpm} / \mathrm{min}$. Scanning electron microscopy was used to observe morphological changes, as described by Wang

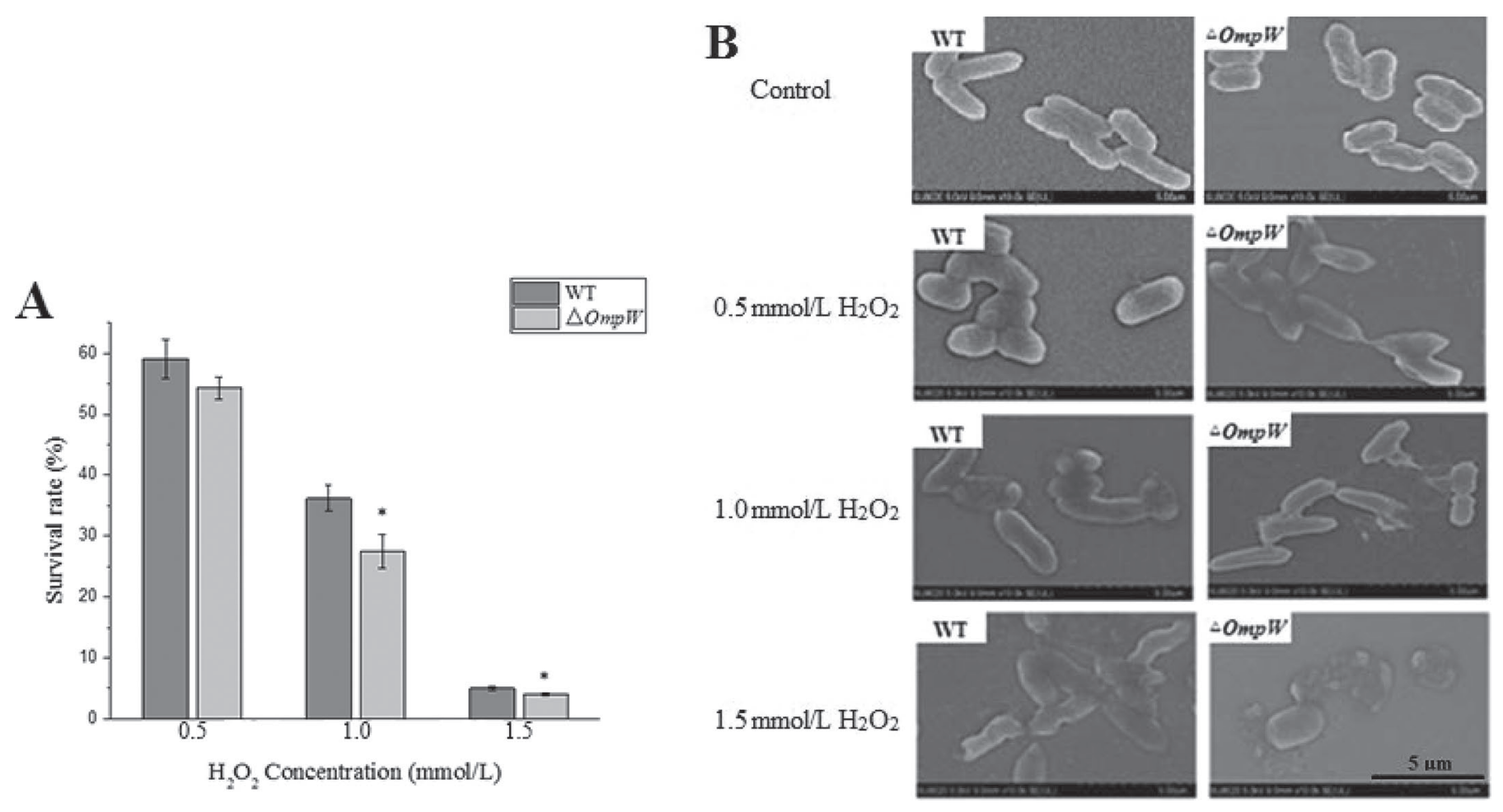

Figure 1. Survival and cellular morphology of Cronobacter sakazakii wild type (WT) and $\Delta O m p W$ under oxidative stress. (A) Survival rates were counted as the number of the hydrogen peroxide-exposed cells divided by the initial cell number multiplied by $100 \%$; data $=$ means \pm SD. ${ }^{*} P<0.05$. (B) Morphological changes of C. sakazakii WT and $\Delta O m p W$ under $0,0.5,1.0$, and $1.5 \mathrm{~m} M \mathrm{H}_{2} \mathrm{O}_{2}$ using scanning electron microscopy. 
et al. (2013) with little modification. From Figure 1B we see that increasing concentrations of $\mathrm{H}_{2} \mathrm{O}_{2}$ caused cellular deformation, leakage of intracellular materials, and damage to the cell membrane or cell wall. In addition, morphological injury was more predominant in $\Delta O m p W$, suggesting OmpW might function as an efflux channel of toxic element (Gil et al., 2009). In E. coli, the porin OmpW participates in the efflux of ethidium multidrug resistance protein E-specific substrates across the outer membrane (Beketskaia et al., 2014). The OmpW expression in Vibrio cholerae was modulated by temperature, salinity, and availability of nutrients or oxygen to adapt to stress (Nandi et al., 2005). In Vibrio alginolyticus, OmpW expression is associated with the tolerance of high sodium concen- trations, whereas $\mathrm{OmpV}$ does not confer tolerance to sodium (Xu et al., 2005).

Bacterial biofilms on surfaces in processing facilities and equipment are the critical factor for cross-contamination in food samples. To determine the biofilm formation under oxidative stress $(0,0.5,1.0$, and $1.5 \mathrm{mM}$ $\mathrm{H}_{2} \mathrm{O}_{2}$ ), crystal violet staining, scanning electron microscopy, and confocal laser scanning microscopy were use as previously described (Ye et al., 2016) with little modification. In Supplemental Table S1 (https://doi .org/10.3168/jds.2018-14643), the biofilms of $\Delta O m p W$ significantly increased at 24, 48, and $72 \mathrm{~h}$ compared

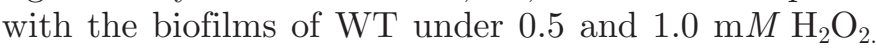
The highest biomasses were formed after $48 \mathrm{~h}$ incubation for 2 strains. To determine the biofilms, the vision

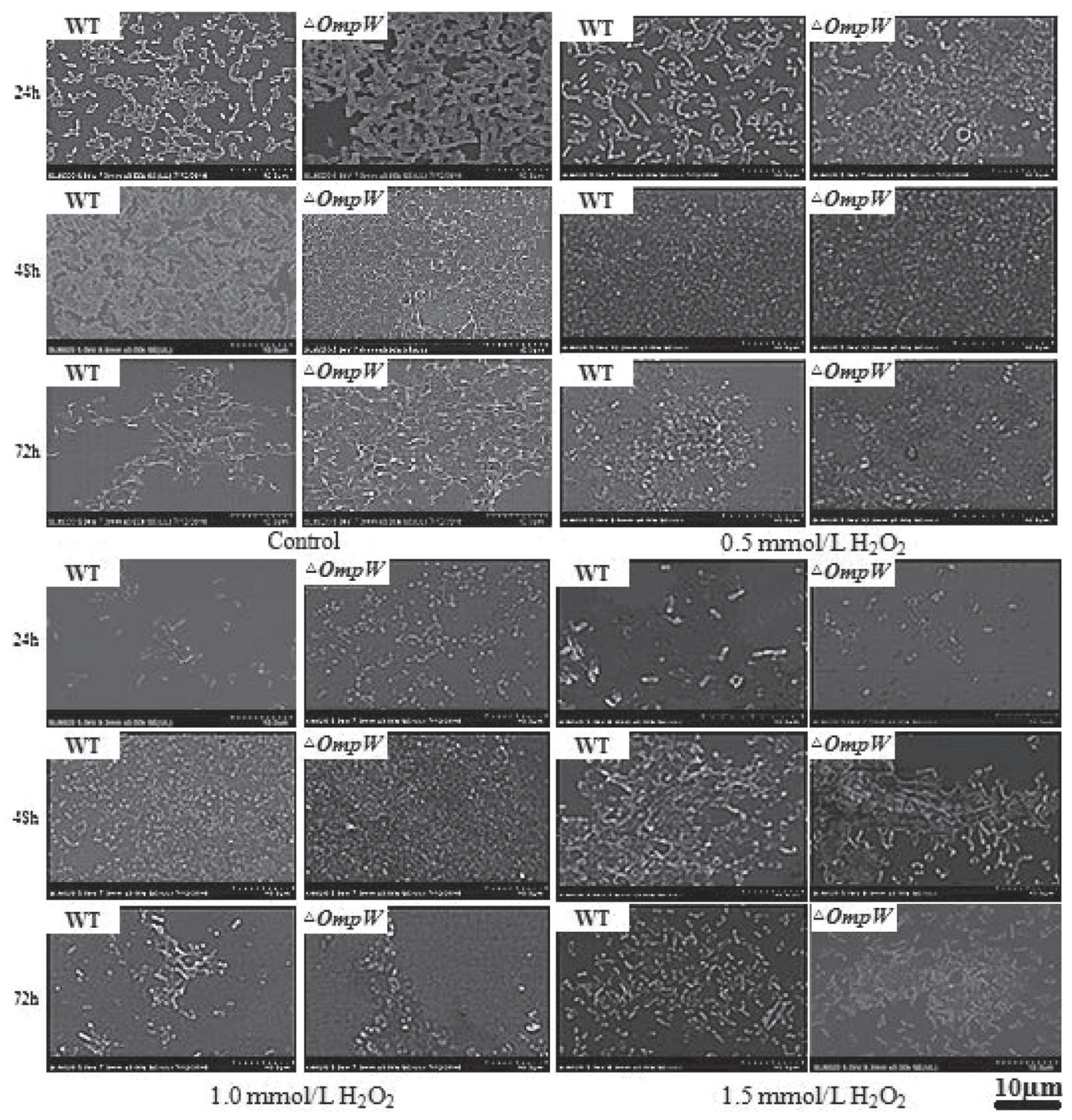

Figure 2. Biofilm formation of Cronobacter sakazakii wild type (WT) and $\Delta O m p W$ under oxidative stress using scanning electron microscopy at 24,48 , and $72 \mathrm{~h}$. 



$1.0 \mathrm{mmol} / \mathrm{L} \mathrm{H}_{2} \mathrm{O}_{2}$



$1.5 \mathrm{mmol} / \mathrm{L} \mathrm{H}_{2} \mathrm{O}_{2}$

Figure 3. Biofilm formation of Cronbacter sakazakii wild type (WT) and $\Delta O m p W$ under oxidative stress using confocal laser scanning microscopy at 24,48 , and $72 \mathrm{~h}$.

standing for overall biofilm from 5 locations was chosen. From Figure 2, mature biofilms were formed at $48 \mathrm{~h}$ and disassembly of biofilms appeared at $72 \mathrm{~h}$. In addition, the biofilm formation was significantly reduced with the increasing concentrations of $\mathrm{H}_{2} \mathrm{O}_{2}$. From Figure 3, the biofilm disassembles at $72 \mathrm{~h}$ and dead cells were more predominant compared with biofilms at $48 \mathrm{~h}$. The OmpW, OmpA, and a type IV pilus were induced in biofilms, and their deletion decreased biofilm formation in Pseudoalteromonas sp. strain D41 (Ritter et al., 2012). Likewise, OmpW expression showed increased abundance in the calcium-induced biofilm in Pseudoalteromonas sp. 1398 (Patrauchan et al., 2005). In $C$. sakazakii, the outer membrane proteins such as OmpC and TolB were potentially involved in biofilm formation using the 2-dimensional electrophoresis method (Ye et al., 2016). Outer membrane protein A (OmpA) and $\mathrm{X}(\mathrm{OmpX})$ were required for invasion and adhesion of Cronobacter sakazakii to Caco-2 and INT-407 cells (Kim et al., 2010). In C. sakazakii ATCC 29544, FliC, FlhA, and FliG contribute to the autoaggregation of cells (Hoeflinger and Miller, 2017). In V. cholerae, matrix proteins including RbmA, Bap1, and RbmC play important roles in biofilm formation (Fong and Yildiz, 2015). In addition, the lectins LecA and LecB, and the Psl-binding matrix protein CdrA in Pseudomonas aeruginosa have also been reviewed to be involved in the biofilm formation (Fong and Yildiz, 2015).

Here, the roles of $O m p W$ in C. sakazakii under oxidative stress were determined. In the future, the complement of $\Delta O m p W$ will be constructed to further confirm the roles of OmpW under other environmental stresses, including desiccation, acid, osmotic, and oxidative stress. 


\section{ACKNOWLEDGMENTS}

We gratefully acknowledge the financial support of the National Key Research and Development Program (2017YFC1601200), National Natural Science Foundation of China (31671951), the Science and Technology Planning Project of Guangdong Province (2016A050502033), Project of Science and Technology in Guangzhou (201604020036), the Fundamental Research Funds for the Central Universities of Hefei University of Technology (PA2017GDQT0018), and State Key Laboratory of Applied Microbiology Southern China Open Foundations, Guangzhou, China (SKLAM004-2015).

\section{REFERENCES}

Alvarez-Ordoñez, A., M. Begley, and C. Hill. 2012. Polymorphisms in rpoS and stress tolerance heterogeneity in natural isolates of Cronobacter sakazakii. Appl. Environ. Microbiol. 78:3975-3984.

Asakura, H., K. Kawamoto, Y. Haishima, S. Igimi, S. Yamamoto, and S. Makino. 2008. Differential expression of the outer membrane protein $\mathrm{W}(\mathrm{OmpW})$ stress response in enterohemorrhagic Escherichia coli O157:H7 corresponds to the viable but non-culturable state. Res. Microbiol. 159:709-717.

Back, K. H., J. W. Ha, and D. H. Kang. 2014. Effect of hydrogen peroxide vapor treatment for inactivating Salmonella Typhimurium, Escherichia coli O157:H7 and Listeria monocytogenes on organic fresh lettuce. Food Control 44:78-85.

Battesti, A., N. Majdalani, and S. Gottesman. 2011. The RpoS-mediated general stress response in Escherichia coli. Annu. Rev. Microbiol. 65:189-213.

Beketskaia, M. S., D. C. Bay, and R. J. Turner. 2014. Outer membrane protein $\mathrm{OmpW}$ participates with small multidrug resistance protein member EmrE in quaternary cationic compound efflux. J. Bacteriol. 196:1908-1914.

Biering, G., S. Karlsson, N. V. C. Clark, K. E. Jonsdottir, P. Ludvigsson, and O. Steingrimsson. 1989. Three cases of neonatal meningitis caused by Enterobacter sakazakii in powdered milk. J. Clin. Microbiol. 27:2054-2056.

Chiang, S. M., and H. E. Schellhorn. 2012. Regulators of oxidative stress response genes in Escherichia coli and their functional conservation in bacteria. Arch. Biochem. Biophys. 525:161-169.

Fong, J. N. C., and F. H. Yildiz. 2015. Biofilm matrix proteins. Microbiol. Spectr. https://doi.org/10.1128/microbiolspec.MB-0004 -2014 .

Gil, F., I. Herna'ndez-Lucas, R. Polanco, N. Pacheco, B. Collao, J. M. Villarreal, G. Nardocci, E. Calva, and C. P. Saavedra. 2009. SoxS regulates the expression of the Salmonella enterica Serovar Typhimurium ompW gene. Microbiology 155:2490-2497.

Gil, F., F. Ipinza, J. Fuentes, R. Fumeron, J. M. Villarreal, A. Aspee, G. C. Mora, C. C. Vasquez, and C. Saavedra. 2007. The OmpW (porin) gene mediates methyl viologen (paraquat) efflux in Salmonella enterica Serovar Typhimurium. Res. Microbiol. 158:529-536.

Gurtler, J. B., J. L. Kornacki, and L. R. Beuchat. 2005. Enterobacter sakazakii: A coliform of increased concern to infant health. Int. J. Food Microbiol. 104:1-34.

Hoeflinger, J. L., and M. J. Miller. 2017. Cronobacter sakazakii ATCC 29544 autoaggregation requires FliC flagellation, not motility. Front. Microbiol. 8:301.
Holý, O., and S. Forsythe. 2014. Cronobacter spp. as emerging causes of healthcare-associated infection. J. Hosp. Infect. 86:169-177.

Huang, Y., and H. Chen. 2011. Effect of organic acids, hydrogen peroxide and mild heat on inactivation of Escherichia coli O157: H7 on baby spinach. Food Control 22:1178-1183.

Jiang, Y., K. Sokorai, G. Pyrgiotakis, P. Demokritou, X. H. Li, S. Mukhopadhyay, T. Jin, and X. T. Fan. 2017. Cold plasma-activated hydrogen peroxide aerosol inactivates Escherichia coli $\mathrm{O} 157: \mathrm{H} 7$, Salmonella Typhimurium, and Listeria innocua and maintains quality of grape tomato, spinach and cantaloupe. Int. J. Food Microbiol. 249:53-60.

Johler, S., R. Stephan, I. Hartmann, K. A. Kuehner, and A. Lehner. 2010. Genes involved in yellow pigmentation of Cronobacter sakazakii ES5 and influence of pigmentation on persistence and growth under environmental stress. Appl. Environ. Microbiol. 76:10531061.

Kim, K., K. P. Kim, J. Choi, J. A. Lim, J. Lee, S. Hwang, and S. Ryu. 2010. Outer membrane proteins a (OmpA) and $\mathrm{X}(\mathrm{OmpX})$ are essential for basolateral invasion of Cronobacter sakazakii. Appl. Environ. Microbiol. 76:5188-5198.

Kim, S., H. Hwang, K.-P. Kim, H. Yoon, D.-H. Kang, and S. Ryu. 2015. hfq plays important roles in virulence and stress adaptation in Cronobacter sakazakii ATCC 29544. Infect. Immun. 83:20892098.

Ling, N., J. M. Zhang, C. S. Li, H. Y. Zeng, W. J. He, Y. W. Ye, and Q. P. Wu. 2018. The glutaredoxin gene, grxB, affects acid tolerance, surface hydrophobicity, auto-aggregation, and biofilm formation in Cronobacter sakazakii. Front. Microbiol. 9:133. https://doi .org/10.3389/fmicb.2018.00133.

Morales, E. H., I. L. Calderón, B. Collao, F. Gil, S. Porwollik, M. McClelland, and C. P. Saavedra. 2012. Hypochlorous acid and hydrogen peroxide-induced negative regulation of Salmonella enterica Serovar Typhimurium ompW by the response regulator ArcA. BMC Microbiol. 12:63.

Nandi, B., R. K. Nandy, A. Sarkar, and A. C. Ghose. 2005. Structural features, properties and regulation of the outer-membrane protein W (OmpW) of Vibrio cholerae. Microbiology 151:2975-2986.

Patrauchan, M. A.. S. Sarkisova, K. Sauer, and M. J. Franklin. 2005. Calcium influences cellular and extracellular product formation during biofilm-associated growth of a marine Pseudoalteromonas sp. Microbiology 151:2885-2897.

Ritter, A., E. Com, A. Bazire, M. D. S. Goncalves, L. Delage, G. L. Pennec, C. Pineau, C. Dreanno, C. Compere, and A. Dufour. 2012. Proteomic studies highlight outer-membrane proteins related to biofilm development in the marine bacterium Pseudoalteromonas sp. D41. Proteomics 12:3180-3192.

van Acker, J., F. De Smet, G. Muyldermans, A. Bougatef, A. Naessens, and S. Lauwers. 2001. Outbreak of necrotizing enterocolitis associated with Enterobacter sakazakii in powdered milk formula. J. Clin. Microbiol. 39:293-297.

Wang, C. Y., C. P. Hsu, H. W. Huang, and B. B. Yang. 2013. The relationship between inactivation and morphological damage of Salmonella enterica treated by high hydrostatic pressure. Food Res. Int. 54:1482-1487.

Xu, C., S. Wang, H. Ren, X. Lin, L. Wu, and X. Peng. 2005. Proteomic analysis on the expression of outer membrane proteins of Vibrio alginolyticus at different sodium concentrations. Proteomics 5:3142-3152.

Ye, Y. W., R. Jiao, J. Gao, H. Li, N. Ling, Q. P. Wu, J. M. Zhang, and X. K. Xu. 2016. Proteins involved in responses to biofilm and planktonic modes in Cronobacter sakazakii. Lebensm. Wiss. Technol. 65:1093-1099.

Ye, Y. W., H. Li, Q. P. Wu, J. M. Zhang, and Y. D. Lu. 2014. The Cronobacter sp. in milk and dairy products: Detection and typing. Int. J. Dairy Technol. 67:167-175. 